\title{
Geometric shape and tensile forces on silo bags for grain storage
}

\author{
Ana Scarabino \\ Computational Fluid Dynamics Group, School of Engineering, National University of La Plata, Argentina
}

\begin{abstract}
This work consists in the analytical derivation and numerical solution of the equation which determines the shape of the section of a silo bag: a long horizontal cylindrical plastic bag filled with either a liquid or a granular material which behaves similarly, exerting normal, but not frictional forces against the wall. The bag is considered inextensible and completely flexible, capable of supporting only tensile loads. These suppositions lead to a secondorder differential equation for the membrane shape, which is normalised and solved, in a way that allows, for any bag with any amount of filling, a simple computation of its geometry, enclosed area and tensile loads. A discussion is included about the effects on the theoretical results of the silo bag tensile deformation.
\end{abstract}

\section{Introduction}

Silo bags are cylindrical plastic bags, hermetically sealed, extensively used for grain storage (Figure 1). They are made of low-density extruded polyethylene, with additives to increase its resistance to UV radiation (INTA, 2009). A bag consists of three layers: an outer white one, which reflects the sunlight, especially ultra-violet radiation, a middle one, and an interior black layer, which minimises the penetration of solar radiation. Only in Argentina, about 45 million tons per year are stockpiled in silo

Correspondence: Ana Scarabino, Computational Fluid Dynamics Group, School of Engineering, National University of La Plata, Argentina.

E-mail: scarabino@ing.unlp.edu.ar

Key words: Grain storage; hydrostatic pressure; membranes; silo-bags.

Acknowledgements: the author is most thankful to Prof. Victoria Vampa and BSc. Daniel Actis for their valuable help at dealing with the Mathematics involved in this work, to Mr. Santiago Calderero for providing the material samples as well as useful information about the silo bags filling process, and to Prof. Mariano Mundo, for carrying out the tensile deformation tests.

Received for publication: 1 May 2019.

Accepted for publication: 26 June 2019.

C Copyright: the Author(s), 2019

Licensee PAGEPress, Italy

Journal of Agricultural Engineering 2019; L:973

doi:10.4081/jae.2019.973

This article is distributed under the terms of the Creative Commons Attribution Noncommercial License (by-nc 4.0) which permits any noncommercial use, distribution, and reproduction in any medium, provid$e d$ the original author(s) and source are credited. bags (Rozadilla et al., 2018). They present advantages as a low cost per ton of stored grain, variable storage capacity, no need of freight during harvest, and good quality of storage, retaining grain moisture, grade and colour (Bartosik et al., 2013).

The motivation for this work is to determine the shape adopted by a silo bag when filled with grain and the forces exerted by the grain on the bag. Although granular materials do not strictly follow the law of hydrostatic pressure $P=P_{o}+\rho g h$ due to friction effects, being their normal stresses usually modelled by Janssen's equation (Janssen, 1895; Schulze, 2019), the hydrostatic pressure model gives a good approximation to their asymptotic behaviour for small storage heights. The pressure distribution of a variety of grains commonly stored in silo bags, as soybean, wheat, corn, rice, barley or sunflower seed, is well modelled by the hydrostatic law at these heights to diameter ratios. Plastic cylindrical bags could also be employed for storage of liquids or could be filled with either water or sand as inflatable dams, for which the present hypothesis is also valid. Although there is increasing bibliography on the physical processes taking part inside the silo bag (Bispo dos Santos et al., 2007; Gaston et al., 2008; Bratsk, 2012), most studies consider an approximate shape, without any geometrical analysis. The closest approaches to derive the true shape of closed horizontal membranes when filled came from the geometries of inflatable dams, as the theoretical analysis of Watson (1985), or the numerical solutions of Abdullah et al. (2005), and others. Some theoretical investigation of analogue problems can also be found in Vassilev et al. (2008).

This work is an attempt to describe the shape, tensile forces, and storage volume per unit length of a flexible horizontal cylindrical membrane filled with a material, which exerts inner hydrostatic pressure. Unlike the mathematical transformations used in Watson (1985) and Vassilev et al. (2008), this analysis will be restrained to Cartesian coordinates, and efforts will be made in order to keep the physical meaning of each parameter clear.

\section{Theoretical analysis}

The hypotheses for this analysis are: i) the bag is completely flexible and inextensible and can only support tensile loads. Its weight is neglected; ii) the bag's longitudinal dimension is considerably larger than its diameter, so the analysis will be two-dimensional for a typical section far from the ends; iii) the filling substance is homogeneous, of constant density (bulk density for grains) and without static friction, so that the hydrostatic pressure gradient holds; iv) the bag shape is symmetrical on a vertical plane, so only one half will be studied.

The bag thickness is around $235 \mu \mathrm{m}$. Typical silo bags diameters range from 6 to $12 \mathrm{ft}$ (approx. 1.83 to $3.66 \mathrm{~m}$ ), and lengths, from 60 to $100 \mathrm{~m}$ (INTA, 2009). Low density polyethylene average mechanical properties in sheet extrusions, reported in the materials database Matweb (MATWEB, 2019), are: density 0.922 $\mathrm{kg} / \mathrm{dm}^{3}$, yield tensile strength $11.2 \mathrm{MPa}$, elongation at break $533 \%$ 
and modulus of elasticity $0.315 \mathrm{GPa}$.

The silo bags, because of their small thickness, present indeed high flexural flexibility (a $60 \mathrm{~m}$ long bag comes completely folded in a box that a single man can handle and two men can lift), and low weight (around $0.22 \mathrm{kgf} / \mathrm{m}^{2}$ ), thus it is realistic to neglect flexural strength and the bag's weight contribution to the inner pressure. However, polyethylene stretches noticeably under tensile loads. Silo bag manufacturers' instructions state that the maximum admissible filling is reached when the bag's tangential stretching deformation is $10 \%$. Suitable marks on the bags allow monitoring its stretching during the filling process. The consequences of not considering this deformation in the theoretical analysis will be examined at the Theoretical results and discussion section.

In this analysis, $X, Y$ will be used for dimensional coordinates, and $x, y$, for normalised non-dimensional coordinates. In order to simplify the equations, the coordinate system will be the one shown in Figure 2. In this way, the pressure distribution inside the bag is

$P=P_{o}+\rho g Y$,

where $P_{o}$ is the minimum pressure imposed by the membrane at the top, $\rho$ is the filling material density (bulk density for grains), $g$ the acceleration of gravity, and $Y$ the vertical coordinate.

Under these assumptions, the membrane tensile load at the top can easily be computed, by considering the equilibrium of forces and moments for half bag, as shown in Figure 2. The horizontal resultant force per unit length of the hydrostatic pressure is $R_{p x}=\frac{\rho g h^{2}}{2}$ applied at $Y=\frac{2}{3} h$. The horizontal resultant force per unit length of the constant pressure $P_{o}$ is $R_{P_{o}}=P_{o h}$, applied at $Y=\frac{h}{2}$.

The weight of the stored grain per unit length is $W$ and the horizontal distance between its centre of gravity and the point of application of the normal force $N$ exerted by the floor (equal and opposite to $W$ ), will be called $e$, so that the normal force and the weight will exert on the filled bag a moment per unit length of value $W e$, which increases the tensile force on the membrane.

Balance of moments is computed about the point of application of the normal force, giving:

$\sum M_{x N}=T_{o} h-W e-P_{o} h \frac{h}{2}-\frac{\rho g h^{2}}{2} \frac{h}{3}=0$
In order to balance moments, the tensile force per unit length $T_{o}$ at the top must be

$T_{o}=\frac{\rho g h^{2}}{6}+\frac{W e}{h}+\frac{P_{o} h}{2}$

The horizontal force on the floor (tensile plus friction), which will not play a role in this analysis, must be:

$T_{h}=\frac{\rho g h^{2}}{3}-\frac{W e}{h}+\frac{P_{o} h}{2}$

Let us now consider a portion of the membrane between the point $(0,0)$ and a point of coordinates $(X, Y)$. The forces acting on it are those shown in Figure 3.

Force balances lead to the following equations:

In the horizontal direction:

$-T_{o}+T_{x}+P_{o} Y+\int_{0}^{Y} \rho g Y d Y=0 \Rightarrow T_{x}=T_{o}-P_{o} Y-\frac{\rho g Y^{2}}{2}$

Considering eq. (3):

$T_{x}=\frac{\rho g h^{2}}{6}+\frac{W e}{h}+\frac{P_{o} h}{2}-P_{o} Y-\frac{\rho g Y^{2}}{2}$

In the vertical direction:

$\int_{0}^{X}\left(P_{o}+\rho g Y\right) d X-T_{y}=0 \Rightarrow T_{y}=P_{o} X+\int_{0}^{X} \rho g Y d X$

By applying the hypothesis that the membrane only supports traction loads, it follows that the resultant load direction must be tangent to the membrane shape, defined by the curve $X(Y)$. So, at any point,

$\frac{T_{x}}{T_{y}}=\frac{d X}{d Y}$

So, we obtain:

$\frac{T_{x}}{T_{y}}=\frac{\left(\frac{\rho g h^{2}}{6}+\frac{P_{o} h}{2}+\frac{W e}{h}\right)-P_{o} Y-\frac{\rho g Y^{2}}{2}}{P_{o} X+\int_{0}^{X} \rho g Y d X}=\frac{d X}{d Y}$


Figure 1. Silo bags. 
This equation can be rearranged as

$$
\frac{\left(\frac{\rho g h^{2}}{6}+\frac{P_{o} h}{2}+\frac{W e}{h}\right)-P_{o} Y-\frac{\rho g Y^{2}}{2}}{\frac{d X}{d Y}}=P_{o} X+\int_{0}^{X} \rho g Y d X
$$

By taking derivatives with respect to $Y$ at both sides, one obtains for $X(Y)$ :

$$
\frac{-\left(\frac{\rho g h^{2}}{6}+\frac{P_{o} h}{2}+\frac{W e}{h}\right) \frac{d^{2} X}{d Y^{2}}-P_{o} \frac{d X}{d Y}+P_{o} Y \frac{d^{2} X}{d Y^{2}}-\rho g Y \frac{d X}{d Y}+\rho g \frac{Y^{2}}{2} \frac{d^{2} X}{d Y^{2}}}{\left(\frac{d X}{d Y}\right)^{2}}=P_{\circ} \frac{d X}{d Y}+\rho g Y \frac{d X}{d Y}
$$

This can be rearranged to an ordinary nonlinear differential equation:

$$
\begin{gathered}
{\left[\frac{Y^{2}}{2}+\frac{P_{o}}{\rho g} Y-\left(\frac{h^{2}}{6}+\frac{P_{o}}{\rho g} \frac{h}{2}+\frac{W e}{\rho g h}\right)\right] \frac{d^{2} X}{d Y^{2}}-\left(\frac{P_{o}}{\rho g}+Y\right) \frac{d X}{d Y}=\left(\frac{P_{o}}{\rho g}+Y\right)\left(\frac{d X}{d Y}\right)^{3}} \\
0 \leq Y \leq h
\end{gathered}
$$

In order to get a non-dimensional equation, characteristic lengths must be defined for normalising $Y$ and $X$. The natural scale for $Y$ is the bag height, $h$; for $X$ we will choose the membrane diameter, $D$, constant for all possible heights $h$. In this way, the quotient $D / h$ (or its reciprocate, $h / D$ ) will be a parameter, which controls the membrane aspect ratio for different levels of filling. By multiplying eq. (12) by $1 / D$ and rearranging terms we obtain for the nondimensional coordinates $x, y$, the following equation and nondimensional parameters:

$$
\begin{aligned}
& \left(\frac{y^{2}}{2}+P y-M\right) \frac{d^{2} x}{d y^{2}}-(P+y) \frac{d x}{d y}=\beta^{2}(P+y)\left(\frac{d x}{d y}\right)^{3} \\
& 0 \leq y \leq 1 \\
& \text { with } \quad x=\frac{X}{D} ; \quad y=\frac{Y}{h} ; \quad \beta=\frac{D}{h} ; \quad P=\frac{P_{o}}{\rho g h} ; \quad M=\frac{1}{6}+\frac{1}{2} P+\frac{W e}{\rho g h^{3}}
\end{aligned}
$$

We will rewrite our equation using primes to indicate derivatives:

$$
\begin{gathered}
\left(\frac{y^{2}}{2}+P y-M\right) x^{\prime \prime}-(P+y) x^{\prime}=\beta^{2}(P+y)\left(x^{\prime}\right)^{3} \\
0 \leq y \leq 1
\end{gathered}
$$

Since only derivatives of $x(y)$ are present in eq. (14), this second order equation can be written as a first order equation for $u=x$ ':

$$
\begin{gathered}
\left(\frac{y^{2}}{2}+P y-M\right) u^{\prime}-(P+y) u=\beta^{2}(P+y) u^{3} ; \\
u=x^{\prime}(y)
\end{gathered}
$$

This differential equation was solved analytically with Wolfram ${ }^{\circledR}$ Mathematica (https://reference.wolfram.com/language/howto/SolveADifferentialEquation.html), giving the general solution for $u(y)$ :

$$
u(y)=\frac{\alpha}{2 \beta} \frac{M-P y-\frac{y^{2}}{2}}{\sqrt{\left(1-\alpha^{2} M^{2}\right)+\left(2 \alpha^{2} M P\right) y+\left(\alpha^{2}\left(M-P^{2}\right)\right) y^{2}-\alpha^{2} P y^{3}-\frac{\alpha^{2}}{4} y^{4}}}
$$

where $\alpha=2 \beta e^{C}$, being $\mathrm{C}$ an integration constant to be determined by boundary conditions.

Physical conditions impose that the derivative of $x(y)$ grows to infinity (horizontal tangent) at $y=0$ (top of the membrane) and $y$ $=1$ (floor). The first condition will be met if the divisor at eq. (16) is zero at $y=0$, which implies that:

$$
1-\alpha^{2} M^{2}=0 \Rightarrow M=\frac{1}{\alpha}
$$

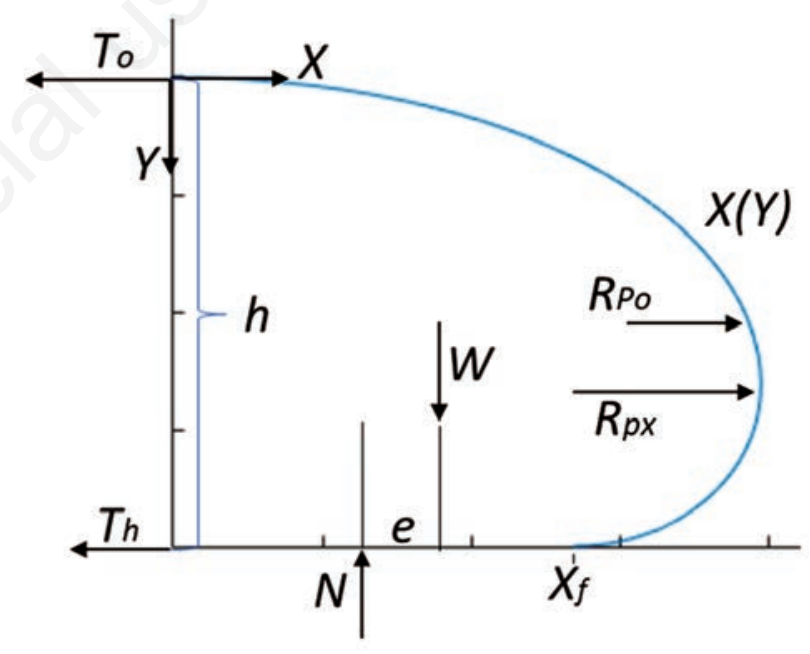

Figure 2. Sketch of coordinates and forces.

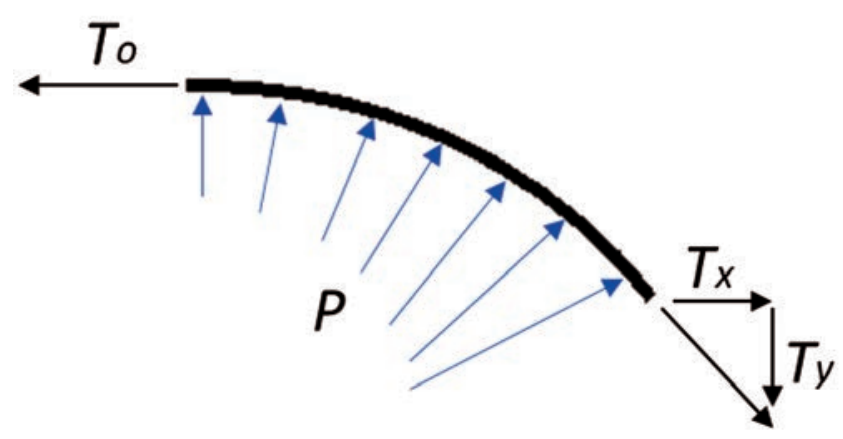

Figure 3. Forces on a segment of the membrane. 
To meet both the first and the second condition, we also need that

$2 \alpha^{2} M P+\alpha^{2}\left(M-P^{2}\right)-\alpha^{2} P-\frac{\alpha^{2}}{4}=0$

By considering eq. (17):

$2 \frac{P}{\alpha}+\frac{1}{\alpha}-P^{2}-P-\frac{1}{4}=0 \Rightarrow \alpha=\frac{2 P+1}{P^{2}+P+\frac{1}{4}} \Rightarrow \alpha=\frac{2}{P+\frac{1}{2}}$

Let us consider now eqs. (17), (19), and the definition of $M$ at eq. (13)

$M=\frac{1}{\alpha}=\frac{P+\frac{1}{2}}{2}=\frac{1}{6}+\frac{P}{2}+\frac{W e}{\rho g h^{3}}$

From this relation, it follows that

$\frac{W e}{\rho g h^{3}}=\frac{1}{12}$

That is, the normalised moment generated between the filling material weight and the floor normal force is constant, and the dimensional moment depends only on the filling height $h$ and the material density, $\rho$.

These results allow writing $u=x^{\prime}(y)$ as a function of only $\beta$ and $P$ :

$u(y)=x^{\prime}(y)=\frac{1}{2 \beta} \frac{\left(\left(P+\frac{1}{2}\right)-2 P y-y^{2}\right)}{\sqrt{\left(\frac{P}{2}+P^{2}\right) y+\left(\frac{1}{4}+\frac{P}{2}-P^{2}\right) y^{2}-P y^{3}-\frac{1}{4} y^{4}}}$

It can be pointed out at this stage, that $u=x^{\prime}$ is zero at the point of maximum width:

$y_{m}=\sqrt{P^{2}+P+\frac{1}{2}}-P$

Integration of $x^{\prime}(y)$ up to this point will give its $x$ normalised coordinate, $x_{m}\left(y_{m}\right)$.

Analytical solutions for expression of this kind usually involve complex elliptical integrals of the first and second kind. We could not find with Mathematica an analytical expression for the integral of equation (22). We have already imposed that the derivatives will grow beyond limit at $y=0$ and (negative) at $y=1$. Numerical integration is possible, imposing the following physical conditions:

- For symmetry, $x(0)=0$;

- The total length of a half membrane, $L=X(h)+\int_{0}^{h} \sqrt{1+\left(\frac{d X}{d Y}\right)^{2}} d Y$ must be constant.

Integration of eq. (22) leads to the definition of $x(y)$ and, $a f_{l}(P, y) \mathrm{s}$

$x(y)=\frac{1}{2 \beta} \int_{0}^{y}\left(\frac{\left(P+\frac{1}{2}-2 P y-y^{2}\right)}{\sqrt{\left(\frac{P}{2}+P^{2}\right) y+\left(\frac{1}{4}+\frac{P}{2}-P^{2}\right) y^{2}-P y^{3}-\frac{1}{4} y^{4}}}\right) d y=\frac{1}{2 \beta} f_{1}(P, y)$


Figure 4. $\mathrm{P} v s \mathrm{~h} / \mathrm{D}$.

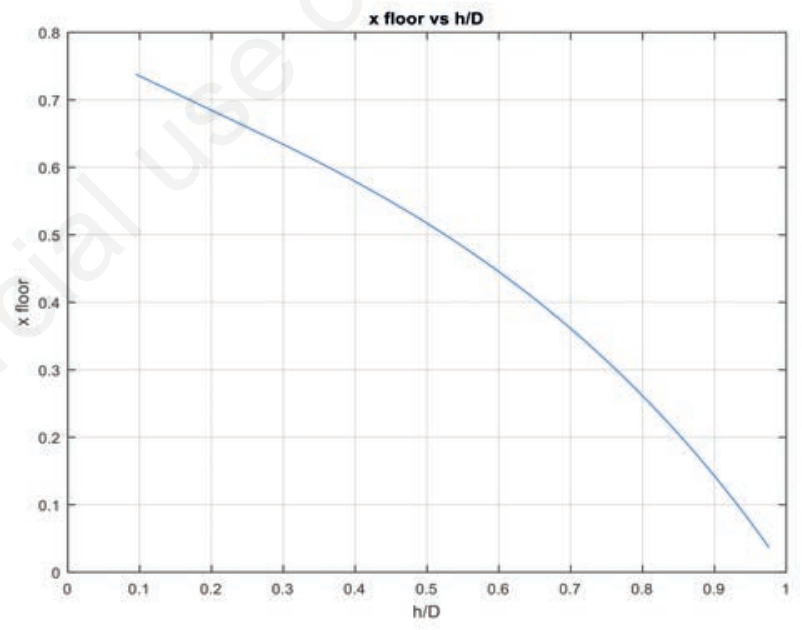

Figure 5. $\mathbf{x} \_$floor $(\mathbf{x}(1)) v s \mathrm{~h} / \mathrm{D}$.

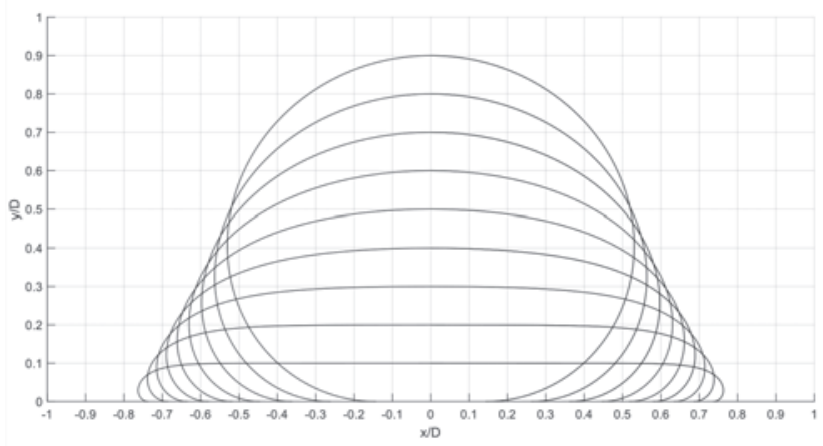

Figure 6. Silo bags shapes for different values of h/D (ordinates at $\mathrm{x} / \mathrm{D}=0$ ). 
For the non-dimensional variables, normalising the expression for the total length, $L$, gives:

$$
\begin{aligned}
& \frac{L}{D}=\frac{\pi}{2}=x(1)+\frac{1}{\beta} \int_{0}^{1} \sqrt{1+\beta^{2} x^{2}} d y \\
& \text { or } \\
& \frac{\pi}{2}=x(1)+\frac{1}{\beta} \int_{0}^{1} \sqrt{1+\frac{1}{4}\left(f_{1}(P, y)\right)^{2}} d y=x(1)+\frac{1}{\beta} f_{2}(P)
\end{aligned}
$$

where is defined in eq. (24). This equation also allows to compute the normalised width at the floor, $x(1)$ as

$$
x(1)=\frac{1}{2 \beta} f_{1}(P, 1)
$$

So, from eqs. (24) and (25) we obtain:

$$
x(1)=\frac{1}{2 \beta} f_{1}(P, 1)=\frac{\pi}{2}-\frac{1}{\beta} f_{2}(P) \Rightarrow \beta=\frac{1}{\pi}\left(f_{1}(P, 1)+2 f_{2}(P)\right)
$$

Function $f_{2}$ was obtained by numerical integration. Plots of $\beta v s$ $P$ and $x(1)$ vs $\beta$ (Figures 4 and 5) show that different parameters tend to their theoretical limits: for $P$ 's limit zero, the membrane is completely flat and folded: $h / D$ is zero and $x(1)$ approaches the

limit value of $\frac{\pi}{4} \approx 0.7854$ (half the length of half a membrane of diameter 1). For $h / D$ reaching its upper limit, $1, P$ tends to infinity, the membrane shape approaches a circumference and $x(1)$ goes to zero.

\section{Theoretical results and discussion}

We are now able, through eq. (27), to determine the aspect ratio $\beta$ as a function of $P$, and then, the normalised function $x(y)$ through numerical resolution of equation (24), the normalised coordinates $\left(x_{m}, y_{m}\right)$ of the point of maximum width and the normalised length lying on the floor, $x_{f}=x(1)$.

Figure 6 shows silo bag shapes for ratios $h / D=1 / \beta$ ranging from 0.1 to the theoretical limit of 1 . The value of parameter $h / D$ for each curve is that of the ordinate at $x=0$.

From eq. (3) and (21) follows that the normalised value of $T_{o}$ is:

$$
\frac{T_{o}}{\rho g h^{2}}=\frac{1}{4}+\frac{P}{2}
$$

Or, considering that $\beta=D / h$,

$$
\frac{T_{o}}{\rho g D^{2}}=\frac{1}{\beta^{2}}\left(\frac{1}{4}+\frac{P}{2}\right)
$$

Values of $\frac{T_{o}}{\rho g D^{2}} v s h / D$ are shown in Figure 7. As the graph shows, it grows very slowly for small heights and it increases considerably for heights above $80 \%$ of the membrane diameter, tending to infinity when $h / D$ approaches 1 .

The physical characteristics of the problem (membrane tensile force supporting a pressure difference) lead to the condition that the tensile force per unit length, T, must be constant along the membrane (Watson, 1985). This fact can be verified by computing the horizontal and vertical components of the tensile force through equations (5) and (7), and their resultant force. So, equations (28) and (29) hold for the whole membrane length at any point, except those in contact with the floor, where friction forces can alter the tensile tangential loads.

The area enclosed by the bag section, $A$, equals the volume of grain per unit length of the silo bag which is stored at a given level of filling. The normalised area $A^{*}=A /(2 D h)$ is given by

$$
A^{*}=\frac{A}{2 D h}=\int_{0}^{1} x(y) d y
$$

Factor 2 is included because the integral at the right side of equation (30) computes only one half of the silo bag section normalised area. A more convenient way to evaluate the silo bag filled section, that is, the stored volume per meter of length, is to evaluate the quotient between the area enclosed by the membrane and the maximum theoretical section, $\pi D^{2} / 4$ then:

$\frac{A}{A_{\max }}=\frac{2 D h \int_{0}^{1} x(y) d y}{\pi D^{2} / 4}=\frac{8}{\pi \beta} \int_{0}^{1} x(y) d y$

Figure 8 shows this ratio $v_{s} h / D=1 / \beta$.

At this point it becomes necessary to discuss the effect of the material stretching, which was neglected in the theoretical analysis. As said, manufacturers' instructions state that the maximum recommended filling should produce a stretching deformation no larger than $10 \%$. Considering the reported material properties and
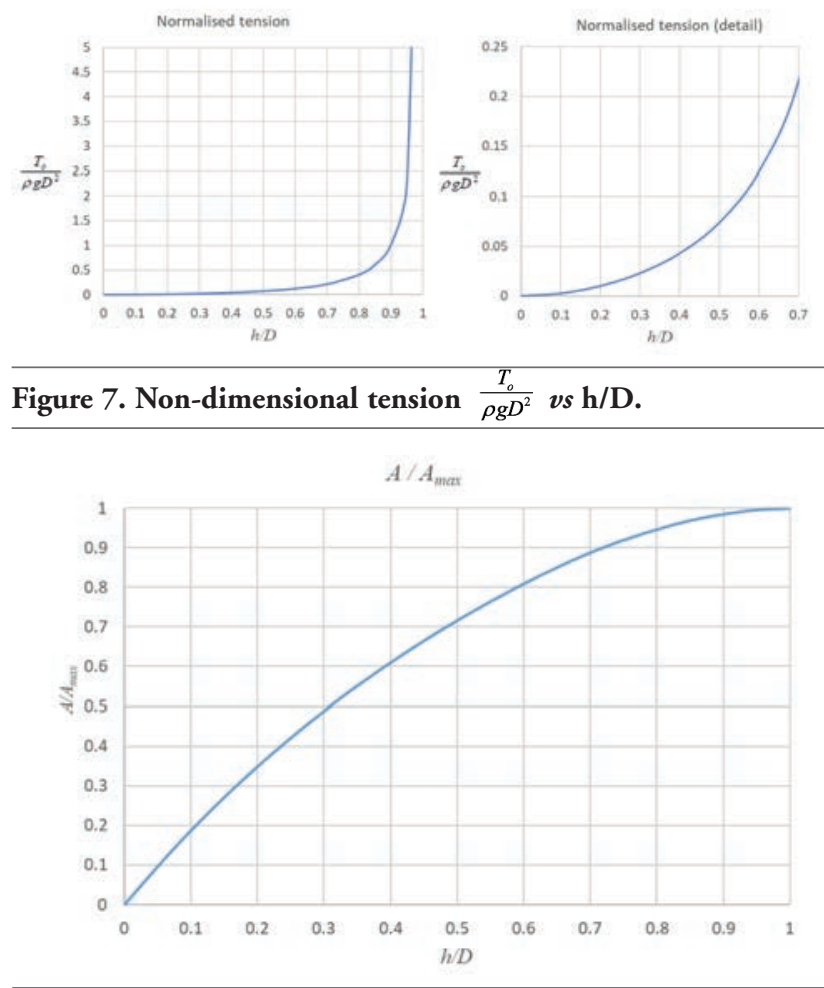

Figure 8. Area/Maximum Area $v s$ h/D. 
thickness, this elongation implies that the material is supporting its yield stress, but with plastic deformations far below break (which occurs at an average 533\% strain).

The theoretical result that tension is constant along the bag perimeter (except for the portion on the floor) will not be altered, in consequence, the elongation is expected to be uniform, and the geometrical shape, to remain approximately the same, but with its dimensions scaled in a factor 1.1. The enclosed area can then be expected to increase by a maximum factor of 1.21 , although floor friction will reduce this value.

Tensile deformation tests of three samples of a silo bag material were performed with an Instron Tensile Tester at the Mechanical Tests Laboratory at the National University of La Plata School of Engineering. Since only three samples were taken, and all from the same bag piece, it is pointed out that the results have no statistical significance, and, in consequence, tension-elongation curves will not be reported in this work. Nevertheless, these measurements will be used to carry out some approximate estimations, which can be enhanced with more accurate values of the material yield stress. To get a $10 \%$ elongation - well in the domain of plastic deformation in the tests- the average measured tensile force per unit width was near $3200 \mathrm{~N} / \mathrm{m}$ (considering a thickness - which was not measured - of $0.235 \mu \mathrm{m}$, this gives a yield stress of 13.6 $\mathrm{MPa}$, whereas the reported average value at MATWEB, 2019, is 11.2 MPa). For a typical $9 \mathrm{ft}(2.743 \mathrm{~m})$ diameter silo bag, filled with grain of bulk density $600 \mathrm{~kg} / \mathrm{m}^{3}$, the normalised tension $\frac{T_{o}}{\rho g D^{2}}$ is 0.072 , which corresponds to a height/diameter ratio of 0.5 (Figure 7), a theoretical (inextensible) enclosed area of $72 \% A_{\max }$, (Figure 8) and a real enclosed area, considering elongation, which can be estimated in roughly $85 \% A_{\max }$. In this example, this gives a storage volume per unit length of approx. $5 \mathrm{~m}^{3} / \mathrm{m}$ or, in mass, approximately $3000 \mathrm{~kg} / \mathrm{m}$. The maximum width (Figure 9) is twice $0.67 \mathrm{D}$, so the bag height to width ratio is $0.5 /(2 * 0.67)=0.37$. Filled silo bags shown in Figure 1 are indeed close to this ratio.

\section{Experiments}

Some simple homemade experiments were carried out with a long cylindrical common polyethylene bag of $D=135 \mathrm{~mm}$, filled first with water (Figure 10) and secondly with sand of measured density $1.59 \mathrm{~kg} / \mathrm{dm}^{3}$. The bag was set on a carefully levelled table. For different amounts of filling, two wood prisms were placed vertically at both sides, in tangential contact with the bag. The distance between them, measured with a standard metric tape, equalled the bag maximum width, $2 X_{m}$. The vertical distance from the bag contact point to the blocks base is, in our coordinate system $h-Y_{m}$. The bag height, $h$, was measured placing a rigid levelled horizontal ruler in tangential contact at the top (but not exerting any vertical force), and measuring on the wood prisms its vertical distance to the table.

An Insize Digital Level and Protractor 2178-1 were used to ensure the table level and the vertical and horizontal orientations of the wood prisms and ruler respectively.

The measured values were normalised by dividing them by $D$ and compared with the theoretical values (eqs. 23, 24 and 32).
$\frac{Y_{m}}{D}=\frac{y_{m}}{\beta}=\frac{1}{\beta}\left(\sqrt{P^{2}+P+\frac{1}{2}}-P\right)$

$\frac{X_{m}}{D}=x_{m}=\frac{1}{2 \beta} f_{1}\left(P, y_{m}\right)$

Results can be seen in Figure 9, showing a very close match for both substances, despite the simplicity and uncertainties of the experiment.

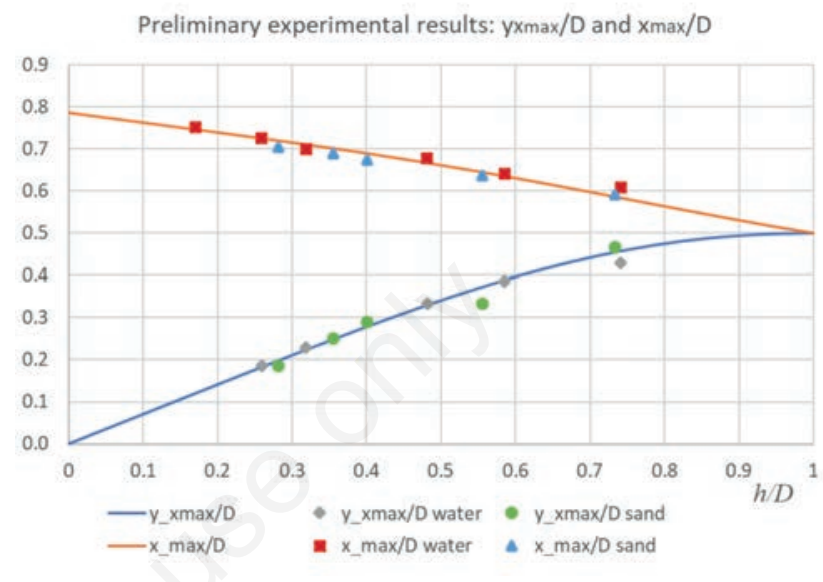

Figure 9. Normalised coordinates of the point of maximum width - theoretical and experimental.

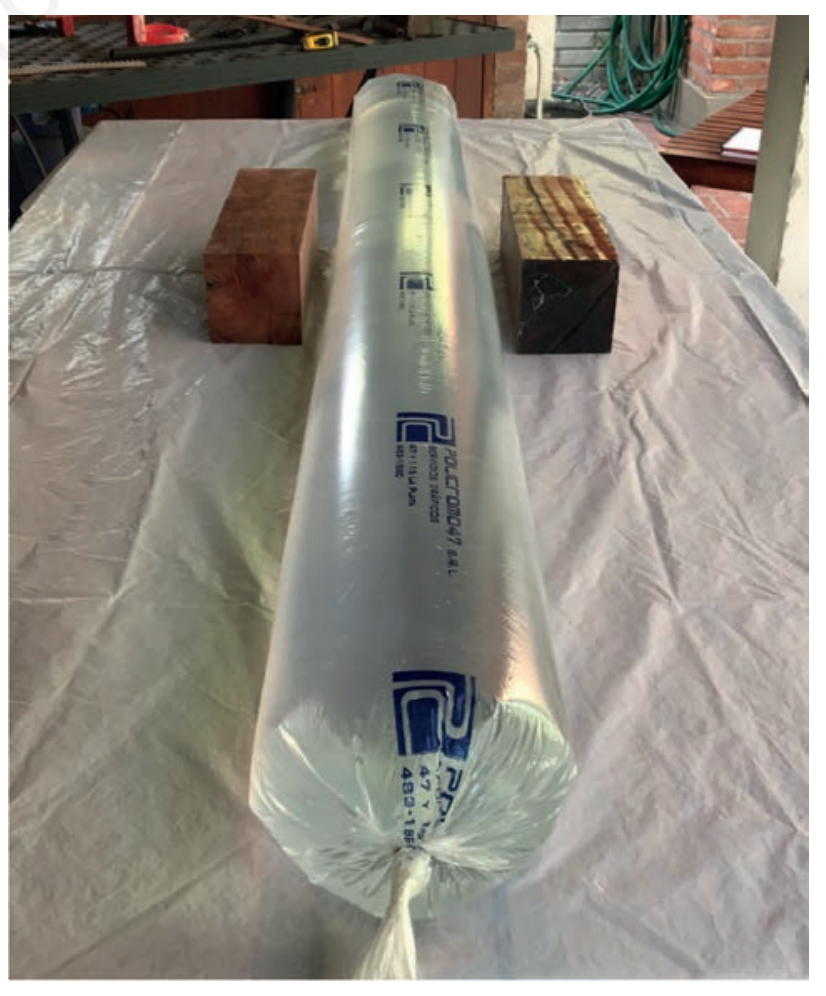

Figure 10. Silo bag model filled with water at $\mathrm{h} / \mathrm{D}=0.74$, and orthogonal wood prisms. These prisms standing vertically were used to measure the bag's width, height and distance to the table of the point of maximum width. 


\section{Conclusions}

A second-order differential equation in Cartesian coordinates was derived for the shape a cylindrical flexible membrane adopts when filled with a liquid or a granular material, which behaves like such. This equation was normalised through two characteristic lengths: the membrane diameter, $D$, and its height when filled, $h$. The normalised equation could be integrated analytically to obtain the first derivative of the membrane shape function, and this was integrated numerically in order to get the final solution. The theoretical computations of some geometrical parameters were validated through simple experimental measurements. For each bag height, these normalised results allow computing parameters as the membrane tensile force, the interior pressure, the maximum width, and the filled cross-section area, which gives the stored volume of cereal per unit length of the silo bag. In the case of extensible materials, the theoretical results can still be used for a first order computation of tensile forces and stretching deformations.

It must be emphasised that the theoretical results for an inextensible material, besides their practical use, can also serve as a benchmark for testing numerical models of static fluid-structure o granular materials - structure interaction.

\section{References}

Abdullah A., Nasser A., Thamer M., Jamalodin N., Abdul G., Essam A. 2005. Behavior of inflatable dams under hydrostatic conditions. Suranaree J. Sci. Technol. 12:1-18.

Bartosik R., Cardoso L., Urcola H., Berruto R. 2013. Economic analysis of storing grain in silobags through a web application. 2013 Conf. on Sustainable Agriculture through ICT
Innovation, Torino, Italy.

Bispo dos Santos S., Aredes Martins M., D’Antonino Faroni L. 2007. Computational study of oxygen infiltration due to damage in the silobag surface. 2007 ASABE International Meeting, Minneapolis, MN, USA.

Bratsk R. 2012. An Inside Look at the Silo-Bag System. Proc 9th. Int. Conf. on Controlled Atmosphere and Fumigation in Stored Products. Antalya, Turkey.

Gaston A., Abalone R., Bartosik R., Rodriguez J.C. 2008. Heat and mass transfer in soybean stored in hermetic plastic bags (silobags). CIGR International Conference of Agricultural Engineering, Foz do Iguazu, Brazil.

INTA (Instituto Nacional de Tecnología Agropecuaria). 2009. Almacenamiento de granos en bolsas plásticas. Resultados de investigación. Ediciones INTA, Córdoba, Argentina [in Spanish].

Janssen H.A. 1895. Getreidedruck in Silozellen. Z. Ver. Dt. Ing. 39:1045-9.

MATWEB. 2009. Material Property Data; June $1^{\text {st }}$ 2019. Available from: http://www.matweb.com/search/DataSheet.aspx? Mat $\mathrm{GUID}=5 \mathrm{c} 9 \mathrm{aca} 1 \mathrm{a} 960945 \mathrm{aa} 8 \mathrm{~d} 9129 \mathrm{f} 8 \mathrm{a} 618 \mathrm{~b} 007$

Rozadilla B., Calzada J., Terré E. 2018. The extensive use of silo bags in Argentina helped to reduce the seasonal variation in grains prices. Noticias Agrícolas 2018. Available from: http://noticiasagricolas.com.ar/?p=15273

Schulze D. 2019. Stresses in Silos. Instituto Nacional de Tecnología Industrial, INTI, Available from: https:/www.i nti.gob.ar/cirsoc/pdf/silos/stresses_silos.pdf

Vassilev V., Djondjorov P., Mladenov I. 2008. Cylindrical equilibrium shapes of fluid membranes. J. Phys A-Math Theor. 41.

Watson R. 1985. A note on the shapes of flexible dams. J. Hydraul. Res. 23:179-94. 